\title{
Bohr's Spectrum of Quantum States in the Atomic Hydrogen Deduced from the Uncertainty Principle for Energy and Time
}

\author{
Stanisław Olszewski \\ Institute of Physical Chemistry, Polish Academy of Sciences, Warsaw, Poland \\ Email: olsz@ichf.edu.pl
}

Received 12 June 2014; revised 8 July 2014; accepted 1 August 2014

Copyright (C) 2014 by author and Scientific Research Publishing Inc.

This work is licensed under the Creative Commons Attribution International License (CC BY).

http://creativecommons.org/licenses/by/4.0/

(c) (i) Open Access

\begin{abstract}
A modified uncertainty principle coupling the intervals of energy and time can lead to the shortest distance attained in course of the excitation process, as well as the shortest possible time interval for that process. These lower bounds are much similar to the interval limits deduced on both the experimental and theoretical footing in the era when the Heisenberg uncertainty principle has been developed. In effect of the bounds existence, a maximal nuclear charge Ze acceptable for the Bohr atomic ion could be calculated. In the next step the velocity of electron transitions between the Bohr orbits is found to be close to the speed of light. This result provides us with the energy spectrum of transitions similar to that obtained in the Bohr's model. A momentary force acting on the electrons in course of their transitions is estimated to be by many orders larger than a steady electrostatic force existent between the atomic electron and the nucleus.
\end{abstract}

\section{Keywords}

Uncertainty Principle for Energy and Time, Bohr's Spectrum of Quantum levels in the Hydrogen Atom

\section{Introduction}

The Bohr model of the hydrogen atom [1] assumed circular trajectories for electrons circulating about the atomic nucleus and the motion along these trajectories has been quantized. In effect definite radii of the orbits

$$
r_{n}=\frac{n^{2} \hbar^{2}}{m e^{2}}
$$

How to cite this paper: Olszewski, S. (2014) Bohr's Spectrum of Quantum States in the Atomic Hydrogen Deduced from the Uncertainty Principle for Energy and Time. Journal of Modern Physics, 5, 1264-1271.

http://dx.doi.org/10.4236/jmp.2014.514127 
and electron velocities on these orbits

$$
v_{n}=\frac{e^{2}}{n \hbar}
$$

could be calculated. From the balance of the electron energy

$$
E_{n}=E_{\text {kin }}\left(v_{n}\right)+E_{\text {pot }}\left(r_{n}\right)=\frac{m v_{n}^{2}}{2}-\frac{e^{2}}{r_{n}},
$$

in which $r_{n}$ and $v_{n}$ are substituted from (1) and (2), a well-known quantum formula

$$
E_{n}=-\frac{m e^{4}}{2 \hbar^{2} n^{2}}
$$

could be obtained. Later it became possible to derive the same $E_{n}$ formula on the basis of a quantummechanical approach [2].

The classical Bohr model stimulated several questions concerning its validity, the main of which was perhaps how the electron - in course of its transition to another orbit—can choose that orbit and become limited in its further motion to it [3] [4]. In other words the problem was concerning not so much separate $E_{n}$, but their differences

$$
\Delta E_{n}=E_{n^{\prime}}-E_{n}=-\frac{m e^{4}}{2 \hbar^{2}}\left(\frac{1}{n^{\prime 2}}-\frac{1}{n^{2}}\right)
$$

for some $n^{\prime}=n+\Delta n$, and the way how (5) can define the atomic spectrum.

The present paper tends to meet this question in case when an approach to the energy spectrum is done on the basis of the uncertainty principle, the essence of which are the energy differences $\Delta E$ applied together with the time intervals

$$
\Delta t=t_{2}-t_{1} .
$$

In fact the principle expressed in the form given by Heisenberg [5]

$$
\Delta E \Delta t>\hbar \text {, }
$$

where $\Delta E$ is a difference of energy met in the quantum process, and has been next objected on many occasions [6]-[8]. The effect of that numerous textbooks on physics or quantum mechanics do not even mention the validity; see e.g. [9]. Nevertheless a modification of (7) into a new formula

$$
2 m c^{2} \Delta E(\Delta t)^{2}>\hbar
$$

could be performed [10]-[12] and we show below that this transformation makes the uncertainty principle suitable in obtainig the atomic spectrum; the Formula (7) is fully ineffective at that point.

But before the spectrum is demonstrated, our aim is to point out that (8) can give also the lower bounds of the space intervals, say that along the Cartesian coordinate $x$ which is

$$
\Delta x=x_{1}-x_{2} .
$$

This result can give a similar lower bound of the time intervals (6) met in the quantum processes.

\section{Lower Bounds of the Position and Time Intervals Derived from the Uncertainty Principle for Energy and Time}

Heisenberg [5] has coupled the observables which are the intervals of the position coordinate and that of momentum in the uncertainty relations similar to that given in (7) for the energy and time. Mathematically this is expressed by the formula

$$
\Delta p_{x} \Delta x>\hbar
$$

on condition that only the $x$-coordinates of the particle momentum and position are taken into account. Though the observables of a pair in (10) enter the formalism on an approximately equal footing, their experimental background can be much different. For example the momentum of a particle is usually much more accurately 
defined than its position. A similar property concerns the energy intervals which are easier accessible than those of time, but - contrary to (7) - the mutual position of $\Delta E$ and $\Delta t$ in (8) is evidently different.

The Relations (10) and (7) imply no limits for $\Delta p_{x}, \Delta x$, as well as $\Delta E$ and $\Delta t$, excepting for their sign. This property comes out from the Formula [13]

$$
\begin{gathered}
p_{x}=\frac{m v_{x}}{\sqrt{1-\frac{v_{x}^{2}}{c^{2}}}}, \\
E=c \sqrt{p_{x}^{2}+m^{2} c^{2}},
\end{gathered}
$$

in which the particle velocity $v_{x}$ is directed along the axis $x$. Both $p_{x}$ and $E$ can rise up unlimitedly with an increase of

$$
v_{x}<c
$$

towards its limit of the light velocity $c$. In effect, in order to satisfy (10) and (7) for arbitrarily large $\Delta p_{x}$ and $\Delta E$, the intervals

$$
\Delta x
$$

and

$$
\Delta t
$$

entering the (10) and (7) should tend to zero.

But this property has been objected already very soon after the the birth of the Heisenberg uncertainty relations [5]. The objections denying the arbitrary small $\Delta x$ and $\Delta t$ have been raised on both the experimental and theoretical footing [14]-[17]. In fact these references proposed that there exists a minimal $\Delta x$ equal to

$$
\Delta x_{0}=\frac{h}{m c}
$$

and a minimal $\Delta t$ equal to

$$
\Delta t_{0}=\frac{h}{m c^{2}},
$$

where $m$ is the rest mass of a particle.

In order to derive (16) from (8) let us assume that $\Delta E$ is the excitation energy of a free particle from the level at $E=0$ to the level of $E=\Delta E$. In this case

$$
\Delta E=\frac{p_{x}^{2}}{2 m}
$$

where $p_{x}$ is the electron momentum.

On the other hand we have

$$
p_{x}=m v_{x}=m \frac{\mathrm{d} x}{\mathrm{~d} t} \cong m \frac{\Delta x}{\Delta t}
$$

which is the effect of one of the Hamilton equations:

$$
\frac{\mathrm{d} x}{\mathrm{~d} t}=\frac{\partial(\Delta E)}{\partial p_{x}}=\frac{p_{x}}{m} .
$$

A transformation of (19) gives

$$
\Delta t=\frac{m}{p_{x}} \Delta x .
$$

This formula, substituted together with (18) into (8), gives:

$$
2 m c^{2} \frac{p_{x}^{2}}{2 m} \frac{m^{2}}{p_{x}^{2}}(\Delta x)^{2}=m^{2} c^{2}(\Delta x)^{2}>\hbar^{2}
$$


or

$$
m c \Delta x>\hbar \text {. }
$$

Therefore we obtain a condition for the smallest $\Delta x$ equal to

$$
\Delta x_{0}=\frac{\hbar}{m c} .
$$

This expression differs from (16) solely by a factor of $2 \pi$. The smallest $\Delta t$, labelled by $\Delta t_{0}$, is obtained from (22) by requirement that the distance $\Delta x_{0}$ is travelled with a maximal speed close to $c$. Therefore

$$
\Delta t_{0}=\frac{1}{c} \Delta x_{0}=\frac{\hbar}{m c^{2}} .
$$

The result in (23) differs from that in (17) solely by the factor of $2 \pi$.

It can be noted that $\Delta t_{0}$ in (23) is only a half of the time interval

$$
T_{0}=\frac{2 \hbar}{m c^{2}}
$$

required to transform the kinetic energy of a particle motion into the energy of the electromagnetic radiation [18].

\section{Discussion}

The lower bounds of $\Delta x$ and $\Delta t$ obtained in (22) and (23) can lead-respectively with the aid of (10) and (8) to the upper bounds of the intervals $\Delta p_{x}$ and $\Delta E$. In particular from (10) and (22) we obtain

$$
\left(\Delta p_{x}\right)_{\max }>\frac{\hbar}{\Delta x_{0}}=\frac{\hbar}{\hbar} m c=m c,
$$

and from (8) and (23):

$$
(\Delta E)_{\max }>\frac{\hbar^{2}}{2 m c^{2}\left(\Delta t_{0}\right)^{2}}=\frac{\hbar^{2}}{2 m c^{2} \hbar^{2}}\left(m c^{2}\right)^{2}=\frac{1}{2} m c^{2} .
$$

If instead of (8) the Heisenberg uncertainty relation (7) together with (23) is applied, the result for the upper bound of $\Delta E$ is

$$
(\Delta E)_{\max }>\frac{\hbar}{\Delta t_{0}}=\frac{\hbar}{\hbar} m c^{2}=m c^{2} .
$$

We see that the Formulae (25)-(27) for the upper bounds of the intervals of momentum and energy approach the relations which are well known from the classical relativistic mechanics. In the present case, however, they are an effect of the quantum-mechanical uncertainty formulae.

If the limiting values $\Delta t=\Delta t_{0}$ and $\Delta E=\frac{1}{2} m c^{2}$ [see (23) and (26)] are substituted into (8), the uncertainty Relation (8) - in order to be satisfied--should attain its certitude in the form

$$
2 m c^{2} \Delta E(\Delta t)^{2}=\hbar^{2},
$$

for in this case holds the relation

$$
2 m c^{2} \frac{1}{2} m c^{2}\left(\frac{\hbar}{m c^{2}}\right)^{2}=\hbar^{2} .
$$

If the energy change $\Delta E$ is a radiation transfer of energy, so

$$
\Delta E=\hbar \Omega \text {, }
$$

there should exist the formula 


$$
\hbar \Omega_{\text {max }}=\frac{1}{2} m c^{2},
$$

defining a maximal frequency $\Omega=\Omega_{\max }$.

For $m$ equal to the electron mass $m_{e}$ we obtain from (30):

$$
\Omega_{\text {max }}=\frac{m_{e} c^{2}}{2 \hbar}=\frac{9.1 \times 10^{-28}\left(3 \times 10^{10}\right)^{2}}{2 \times 1.05 \times 10^{-27}} \cong 0.4 \times 10^{21} \mathrm{sec}^{-1} .
$$

Roughly a similar result for $\Omega_{\max }$ in a metal has been obtained in [10].

Moreover, for $m=m_{e}$ we have $\Delta x_{0} \sim 0.3 \times 10^{-10} \mathrm{~cm}$ from (22) and $\Delta t_{0} \sim 10^{-21} \mathrm{sec}$ from (23). The lower limits for the free-electron path and electron transition time in metals calculated before [19] fit the $\Delta x_{0}$ and $\Delta t_{0}$ quoted above.

If the orbital radii of a hydrogen-like atom having the nucleus of charge $Z e$ are considered instead of charge $\mathrm{Ze}=\boldsymbol{e}$ alone, the orbital radii become [20] [21]

$$
r_{n}=\frac{n^{2} \hbar^{2}}{m Z e^{2}}
$$

giving the smallest radius $(n=1)$

$$
r_{1}=\frac{\hbar^{2}}{m Z e^{2}} .
$$

According to the result obtained in (22) the smallest possible radius (32) should satisfy the relation

$$
r_{1}=\frac{\hbar^{2}}{m Z e^{2}}=\frac{\hbar}{m c} \text {. }
$$

This gives a limiting condition for the atomic number

$$
Z=\frac{c \hbar}{e^{2}} \cong 137 .
$$

A similar condition can be obtained by considering the shortest time period $T_{c r}$ acceptable for the orbit $n=1$. In this case from [20] and the result in (22) it should be satisfied

$$
T_{c r}=\frac{2 \pi \hbar^{3}}{m Z^{2} e^{4}}=\frac{\hbar}{m c^{2}} .
$$

By applying next the Formula (33a) in (34) we obtain the relation

$$
\frac{2 \pi \hbar^{3}}{m_{e}\left(\frac{c \hbar}{e^{2}}\right) Z e^{4}}=2 \pi \frac{\hbar^{2}}{m Z e^{2} c}=\frac{\hbar}{m c^{2}}
$$

from which, by multiplying the both sides of the last equation in (35) by $c$ and taking into account (32a), we have

$$
2 \pi r_{1}=\frac{\hbar}{m c} .
$$

The sense of (35a) is that in order to obtain from (35a) the same limiting $Z$ as in (33a) we should assume $2 \pi r_{1}$ instead of $r_{1}$ in (32a) as a smallest acceptable distance. This is an expected result if we note that the time formula for $T_{c r}$ involves necessarily the orbit length $2 \pi r_{1}$ in calculations and not $r_{1}$ alone.

\section{The Uncertainty Relation (8) Referred to the Energy Spectrum of the Hydrogen Atom}

Till the present point the electron motion on the orbits has been mainly taken into account. But in calculating the electron transitions between orbits the electron velocity normal to the orbital trajectories has to be considered. 
Let the electron which is on a circular orbit labelled by $n$, so its radius is defined in (1), be promoted to another orbit, for example $n+\Delta n$. An effect of this transition is a change of the orbit radius by the amount

$$
\Delta r_{n}=r_{n+\Delta n}-r_{n}=\left[(n+\Delta n)^{2}-n^{2}\right] \frac{\hbar^{2}}{m e^{2}} \approx 2 n \Delta n \frac{\hbar^{2}}{m e^{2}},
$$

on condition we assume that

$$
\Delta n \ll n,
$$

so the term $(\Delta n)^{2}$ can be henceforth neglected. In course of the electron transition we assume that radiation of velocity $c$ is emitted or absorbed. Roughly the time of transition from $r_{n}$ to $r_{n+\Delta n}$ can be estimated by the absolute value of the expression

$$
\Delta r_{n} \approx c \Delta t_{n} .
$$

The property that the time interval $\Delta t_{n}$ satisfies (38) can be derived from the uncertainty principle (8). In a limiting case of (8) the Formula (8a) is valid from which

$$
\Delta t=\frac{\hbar}{\left(2 m c^{2} \Delta E\right)^{1 / 2}} .
$$

In course of the transition process the electron energy on the orbit is changed by an absolute amount equal to [see (5)]:

$$
\left|\Delta E_{n}\right|=\frac{m e^{4}}{2 \hbar^{2}}\left|\frac{1}{(n+\Delta n)^{2}}-\frac{1}{n^{2}}\right| \approx \frac{m e^{4}}{2 \hbar^{2}}\left|\frac{(n+\Delta n)^{2}-n^{2}}{n^{4}}\right| \approx \frac{1}{n^{3}}|\Delta n| \frac{m e^{4}}{\hbar^{2}} .
$$

If we put

$$
\left|\Delta E_{n}\right|=\Delta E,
$$

we obtain from (39) and (40) the formula

$$
\Delta t=\Delta t_{n} \cong \frac{\hbar}{\left(2 m c^{2}\right)^{1 / 2}} \frac{\hbar}{m^{1 / 2} e^{2}} \frac{n^{3 / 2}}{|\Delta n|^{1 / 2}}=\frac{\hbar}{m c e^{2}} \frac{n^{3 / 2}}{2^{1 / 2}|\Delta n|^{1 / 2}} .
$$

For large $n$ the time interval $\Delta t_{n}$ is found to be a short amount of the electron circulation time period

$$
T_{n}=\frac{2 \pi n^{3} \hbar^{3}}{m e^{4}}
$$

on the orbit $n \quad$ [20]; $Z=1$. For from (43) and (42) we have:

$$
\frac{T_{n}}{\Delta t_{n}}=\frac{2 \pi n^{3} \hbar^{3}}{m e^{4}} \frac{m e^{2} c}{\hbar^{2}} \frac{2^{1 / 2}|\Delta n|^{1 / 2}}{n^{3 / 2}}=2^{3 / 2} n^{3 / 2} \pi|\Delta n|^{1 / 2} \frac{\hbar c}{e^{2}}=2^{3 / 2} n^{3 / 2} \pi|\Delta n|^{1 / 2} 137,
$$

where the factor of 137 is coming from the constant expression (33a).

A characteristic point is that a substitution of $\Delta r_{n}$ and $\Delta t_{n}$ from (36) and (42), respectively, to the expression of velocity roughly estimated in (38) gives

$$
\frac{\Delta r_{n}}{\Delta t_{n}}=2 n|\Delta n| \frac{\hbar^{2}}{m e^{2}} \frac{m e^{2} c}{\hbar^{2}} \frac{2^{1 / 2}|\Delta n|^{1 / 2}}{n^{3 / 2}}=\frac{2^{3 / 2}|\Delta n|^{3 / 2}}{n^{1 / 2}} c .
$$

For $\Delta n=1$ and $n=8,9,10,11, \cdots$ we obtain

$$
\frac{\Delta r_{n}}{\Delta t_{n}}=c,\left(\frac{8}{9}\right)^{1 / 2} c,\left(\frac{8}{10}\right)^{1 / 2} c,\left(\frac{8}{11}\right)^{1 / 2} c \cdots
$$

The largest velocity (45) coming from $\Delta n=1$ and $n=1$ is $2^{3 / 2} c \cong 2.8 c$.

If an approximate time interval 


$$
\Delta t_{n} \approx \frac{\Delta r_{n}}{c} \approx 2 n|\Delta n| \frac{\hbar^{2}}{m e^{2} c}
$$

is substituted in place of $\Delta t$ into the Formula (8a) we obtain

$$
2 m c^{2} \Delta E(2 n \Delta n)^{2}\left(\frac{\hbar^{2}}{m e^{2} c}\right)^{2}=\hbar^{2}
$$

from which

$$
\Delta E=\frac{\hbar^{-2} m^{2} e^{4} c^{2}}{2 m c^{2}} \frac{1}{(2 n \Delta n)^{2}}=\frac{m e^{4}}{2 \hbar^{2}} \frac{1}{(2 n \Delta n)^{2}} .
$$

This expression becomes equal to that obtained from the Bohr theory in (40) on condition

$$
\frac{1}{(2 n \Delta n)^{2}}=\frac{2|\Delta n|}{n^{3}}
$$

or

$$
|\Delta n|^{3}=\frac{1}{8} n
$$

are satisfied.

In effect a proper choice of $\Delta n$ for a given $n$ provides us with the relation

$$
\left|\Delta E_{n}\right|=\Delta E \text {. }
$$

Certainly, the $\Delta n$ can approach an integer number only at a special $n$.

\section{The Momentary Force Acting on an Electron in Case of Its Transition between the Orbits}

A stationary force acting on the electron of the hydrogen atom is a well-known electrostatic force of the electron attraction by the nucleus. This force is assumed by Bohr to be compensated by the centrifugal force of the electron orbital motion, so on an orbit having the index $n$ the electron is submitted to the force

$$
F_{\text {stationary }}=\frac{e^{2}}{r_{n}^{2}}=\frac{m v_{n}^{2}}{r_{n}}=\frac{m^{2} e^{6}}{n^{4} \hbar^{4}} .
$$

Evidently this force is of a stationary character. Our aim is to approach a momentary force acting in course of the electron transition between the orbits.

Since the electron velocity of transition is close to $c$ [see (45)], the electron momentum associated with transition is a product equal approximately to $m c$, where $m$ is the electron mass. The momentary force active in a transition from the orbit $n$ to another orbit $n+\Delta n$ is

$$
F_{\text {mom }} \cong \frac{m c}{\Delta t_{n}}=\frac{m^{2} c^{2} e^{2}}{\hbar^{2}} \frac{2^{1 / 2}|\Delta n|^{1 / 2}}{n^{3 / 2}}
$$

where the transition time $\Delta t_{n}$-assumed to be the time of the action of the force due to the momentum $m c$ possessed approximately by the electron-is that calculated in (42). The force obtained in (53) is much larger than (52) because of the ratio

$$
\frac{F_{\text {stationary }}}{F_{\text {mom }}}=\frac{m^{2} e^{6}}{n^{4} \hbar^{4}} \frac{\hbar^{2}}{m^{2} c^{2} e^{2}} \frac{n^{3 / 2}}{2^{1 / 2}|\Delta n|^{1 / 2}}=\left(\frac{e^{2}}{c \hbar}\right)^{2} \frac{1}{2^{1 / 2} n^{5 / 2}|\Delta n|^{1 / 2}}=\left(\frac{1}{137}\right)^{2} \frac{1}{2^{1 / 2} n^{5 / 2}|\Delta n|^{1 / 2}} .
$$

When the energy relations instead of the forces are taken into account, the force given in (53) is multiplied by the distance $\Delta r_{n}$ travelled upon it, so the energy of transition is

$$
E_{\text {mom }} \cong m c \frac{\Delta r_{n}}{\Delta t_{n}} \approx m c^{2},
$$


on condition the velocity property presented in (38) is unchanged. In this case the energy $E_{\text {mom }}$ in (55) is much larger than $\Delta E$ associated with any transition between the electron orbits.

\section{Summary}

In the first step we have demonstrated on the basis of a modified uncertainty relation between the intervals of energy and time that both an interval of the particle position and that of time can attain some minimal values different than zero. These interval limits differ solely by a factor of $2 \pi$ from those predicted a long time ago on both the experimental and theoretical footing [5]-[7]. When applied to the hydrogen-like atom, the lower bounds of the intervals for the position interval and interval of time lead to an upper bound for the atomic number $Z$ equal to 137. On the other hand the upper bounds of the intervals of momentum and energy, obtained from the lower bounds of $\Delta x$ and $\Delta t$, approach the relations which are well known from the classical relativistic mechanics.

A separate attention has been attached to the velocity calculation for the electron transitions between the orbits. First, with the aid of the uncertainty principle, it has been demonstrated that-for the atomic levels having index $n$ equal to about 10-this velocity is very close to the light speed $c$. Moreover, the energy changes $\Delta E$ which accompany the electron transitions become close to those known from the Bohr's atomic theory. Such result cannot be obtained from the uncertainty Formula (7).

The ratio of the stationary electrostatic force acting on the atomic electron to a momentary force active only in course of the electron transition between the orbits has been estimated in (54).

\section{References}

[1] Bohr, N. (1913) Philosophical Magazine, 26, 1. de Boer, J., Dal, E. and Ulfbeck, O. (1986) The Lesson on Quantum Theory-Niels Bohr Centenary Symposium. North-Holland, Amsterdam.

[2] Schrödinger, E. (1926) Annalen der Physik, 79, 361-376. http://dx.doi.org/10.1002/andp.19263840404

[3] Bohr, N. (1961) Proceedings of the Physical Society, 78, 1083. http://dx.doi.org/10.1088/0370-1328/78/6/301

[4] Tarasov, L.V. (1978) Foundations of Quantum Mechanics. Vyzszaja Szkola Moscow. (in Russian)

[5] Heisenberg, W. (1927) Zeitschrift für Physik, 43, 172-198. http://dx.doi.org/10.1007/BF01397280

[6] Schommers, W. (1989) Space-Time and Quantum Phenomena. In: Schommers, W., Ed., Quantum Theory and Pictures of Reality, Springer, Berlin. http://dx.doi.org/10.1007/978-3-642-95570-9_5

[7] Allcock, G.R. (1969) Annals of Physics, 53, 253-285. http://dx.doi.org/10.1016/0003-4916(69)90251-6

[8] Bunge, M. (1970) Canadian Journal of Physics, 48, 1410-1411. http://dx.doi.org/10.1139/p70-172

[9] Isaacs, A. (1990) Concise Dictionary of Physics. Oxford University Press, Oxford.

[10] Olszewski, S. (2011) Journal of Modern Physics, 2, 1305-1309. http://dx.doi.org/10.4236/jmp.2011.211161

[11] Olszewski, S. (2012) Journal of Modern Physics, 3, 217-220. http://dx.doi.org/10.4236/jmp.2012.33030

[12] Olszewski, S. (2012) Quantum Matter, 1, 127-133. http://dx.doi.org/10.1166/qm.2012.1010

[13] Landau, L.D. and Lifshitz, E.M. (1969) Mechanics. Electrodynamics. Nauka, Moscow. (in Russian)

[14] Ruark, A.E. (1928) Proceedings of the National Academy of Sciences of the United States of America, $14,322$. http://dx.doi.org/10.1073/pnas.14.4.322

[15] Flint, H.E. (1928) Proceedings of the Royal Society A, London, 117, 630. http://dx.doi.org/10.1098/rspa.1928.0025

[16] Flint, H.E. and Richardson, O.W. (1928) Proceedings of the Royal Society A, London, 117, 637. http://dx.doi.org/10.1098/rspa.1928.0026

[17] Jammer, M. (1966) The Conceptual Development of Quantum Mechanics. McGraw-Hill, New York.

[18] Arbab, A.I. and Yassein, F.A. (2012) Journal of Modern Physics, 3, 163-169. http://dx.doi.org/10.4236/jmp.2012.32022

[19] Olszewski, S. (2013) Quantum Matter, 2, 408-411. http://dx.doi.org/10.1166/qm.2013.1072

[20] Sommerfeld, A. (1931) Atombau und Spektrallinien. Vol. 1, 5th Edition, Vieweg, Braunschweig.

[21] Slater, J.C. (1960) Quantum Theory of the Atomic Structure. Vol. 1, McGraw-Hill, New York. 
Scientific Research Publishing (SCIRP) is one of the largest Open Access journal publishers. It is currently publishing more than 200 open access, online, peer-reviewed journals covering a wide range of academic disciplines. SCIRP serves the worldwide academic communities and contributes to the progress and application of science with its publication.

Other selected journals from SCIRP are listed as below. Submit your manuscript to us via either submit@scirp.org or Online Submission Portal.
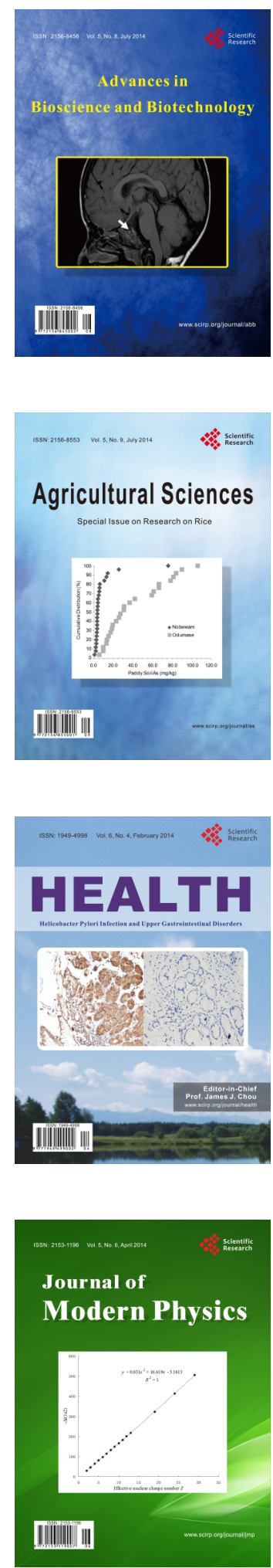
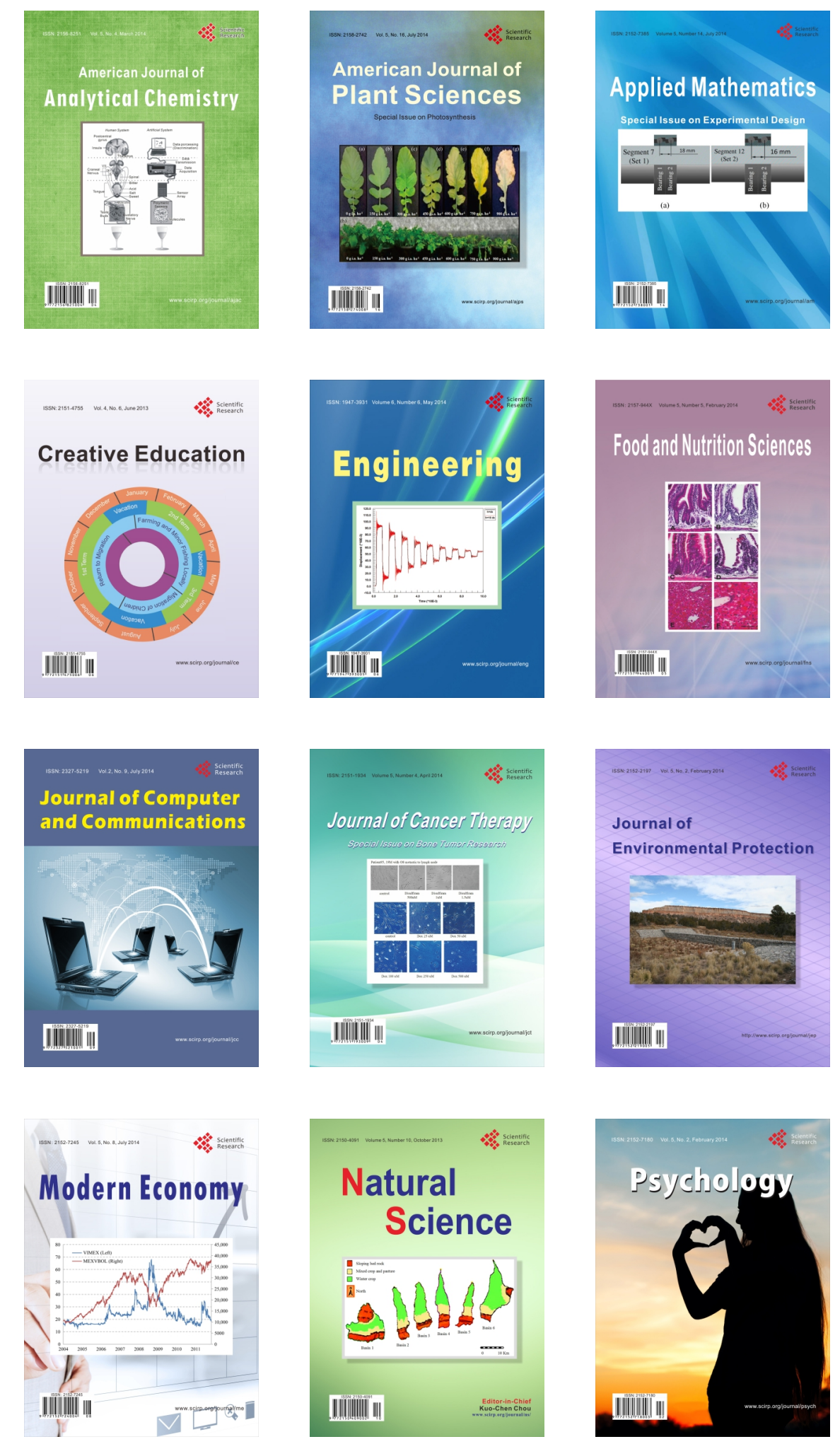